\title{
Vascularização arterial e venosa do linfonodo poplíteo em cães
}

Karina de Senna VILLAR ${ }^{1}$

Wilson Machado de

SOUZA $^{1}$

\section{Correspondência para:}

Rua Clóvis Pestana, 793, Dona Amélia,

16050-680, Araçatuba, SP, Brasil.

wmsouza@fmva.unesp.br.

Recebido para publicação: 20/01/2006 Aprovado para publicação: 29/06/2009

\begin{abstract}
1 - Departamento de Apoio a Produção e Saúde Animal do Curso de Medicina Veterinária da Faculdade de Odontologia da Universidade Estadual Paulista "Julio de Mesquita Filho", Araçatuba-SP
\end{abstract}

\section{Resumo}

O linfonodo poplíteo nos cães situa-se, à direita e à esquerda em um espaço designado fossa poplítea, no terço distal dos músculos bíceps femoral, lateralmente e semitendinoso, medialmente, projetando-se na altura da face caudal das articulações femoro tibial e femoro patelar (articulação do joelho). Neste estudo foram utilizados vinte e cinco cães, machos e fêmeas adultos, procedentes do canil municipal de Araçatuba, São Paulo, cujas capturas foram efetuadas pela defesa sanitária animal desta cidade. Os vasos arteriais destinados a esta estrutura derivam sempre, de ambos os lados, da artéria femoral caudal distal e variam de 10 a 1, com maior freqüência de 2 ( 7 vezes, $28 \%$ ) à direita e de 6 a 2 igualmente com maior freqüência de 2 (8 vezes, 32\%). Tão logo tem-se ainda à direita 5 e 6 ramos (3 vezes, $12 \%), 1,7$ e 10 (1 vez, 4\%). Relativamente às veias derivadas deste linfonodo, convergem sempre para a veia safena lateral, estes vasos oscilam entre 9 e 2 , com maior freqüência de 3 ( 9 vezes, 3,6\%), à direita e de 12 a 2, com maior freqüência de 3 (10 vezes, 40\%) à esquerda. Assim, os demais ramos oriundos da linfonodo poplíteo direito e que se inserem na veia supracitada são em número de 2 e 5 ( 5 vezes, $20 \%$ ), 4 e 6 ( 2 vezes, $8 \%$ ) e 8 e 9 ( 1 vez, $4 \%$ ). Não obstante, ao lado esquerdo verifica-se em número de 2 ramos (6 vezes, 24\%), 4 (4 vezes, 16\%), 6 ( 3 vezes, $12 \%$ ) e finalizando 9 e 12 ramos ( 1 vez, $4 \%$ ). O tamanho do linfonodo poplíteo em cães soropositivos para Leishmaniose Visceral pode encontrar-se entre 7,8 x 3,8 x 6,1 a 50,0 x $20,7 \times 28,5$, em mm, sendo a média 26,18 x 10,5 x 15,97 mm para o direito e $26,98 \times 11,14 \times 15,25 \mathrm{~mm}$ para o esquerdo (referente as medidas dorso-ventral, latero-lateral e crânio-caudal, respectivamente).

\section{Introdução}

O cão constitui-se num elemento de qualidade de vida no meio urbano, onde exerce um papel de substituto de relações sociais pouco satisfatórias, e onde ele é um verdadeiro refúgio contra o isolamento. Como auxiliar do homem, o seu papel tem sido grandemente ampliado, é conhecido o uso do cão como animal de companhia, exercendo, por vezes, papel essencial na vida dos idosos e das crianças, além do caráter terapêutico quando da sua presença nos hospitais ou nos asilos com os deficientes.

Particularizando a espécie canina, o linfonodo poplíteo, situado na fossa ilíaca, é o mais rotineiramente utilizado pelos veterinários na palpação de forma especial quando existe suspeita de afecções relacionadas com o sistema circulatório, como é o caso da Leishmaniose Visceral, zoonose cujos casos têm sido freqüentes na região de Araçatuba. Os linfonodos poplíteos podem variar seu tamanho de $0,5 \mathrm{~cm}$ a $5,0 \mathrm{~cm}^{1}$, na dependência do porte e da raça. A importância deste estudo pode ser avaliada diante das informações observadas por diversos autores Pflug e Calnan 2; Scwarze ${ }^{3}$; Cordeiro e Baracat ${ }^{4}$; Knox e Pflug ${ }^{5}$; Parsons e Romer ${ }^{6}$; Spalding 
e Heath ${ }^{7}$; Hall e Sinnet ${ }^{8}$; Salvador, Pereira e Grande ${ }^{9}$; Salvador et al. ${ }^{10}$; Evans ${ }^{11}$; Budras, Fricke e McCarthy ${ }^{12}$; Sasaki, Pabst e Rothkotter ${ }^{13}$; Belz e Heath ${ }^{14}$ e Barone ${ }^{15}$, cujos dados permitem uma comparação com aqueles que observamos.

\section{Material e Método}

Neste estudo foram utilizados vinte e cinco cães, machos e fêmeas adultos, sem raça definida, com peso corpóreo oscilando entre 10 e 26 quilos, procedentes do canil municipal cujas capturas foram efetuadas pela defesa sanitária animal desta cidade. Estes animais foram convenientemente sedados e eutanasiados mediante anestésicos rotineiramente indicados para esse fim. Todos os animais obtiveram resultado positivo para o exame da Leishmaniose, realizados no Instituto Adolfo Lutz de Araçatuba, através dos métodos de ELISA e Imunofluorescência Indireta. Dessa amostragem, analisamos o total de 25 animais, 15 machos e 10 fêmeas.

Em seguida, foi feita uma laparotomia mediana nesses animais. Desta maneira depois de ampla exposição das vísceras abdominais, à artéria aorta foi isolada e canulada com sonda metálica adaptada à sonda de látex de calibres compatíveis, esta manobra foi realizada no trato deste vaso após a emergência das artérias renais. Através desta cânula foi injetada solução salina a 1\% objetivando uma completa lavagem dos vasos arteriais e venosos correspondentes à região. Após este procedimento, foi injetada na artéria aorta, mediante pressão manual, solução de látex natural diluído em água e corado em vermelho, simultaneamente à injeção da mesma substância corada em azul no trato correspondente da veia cava caudal, injeção complementada por meio de procedimento semelhante através da veia safena.

Em seguida, de cada um dos membros pélvicos os vasos sangüíneos destinados ao linfonodo poplíteo foram dissecados procurando-se estabelecer suas origens e trajetos. As estruturas foram medidas, sua morfologia e observações descritas. As preparações assim trabalhadas foram esquematizadas e fotografadas objetivando registrar a sintopia do referido linfonodo, associando estas relações com sua vascularização. Fotografias e esquemas foram confeccionados visando a documentação e ilustração do trabalho.

\section{Resultados}

O linfonodo poplíteo nos cães situase, à direita e à esquerda em um espaço designado fossa poplítea, no terço distal dos músculos bíceps femoral, lateralmente e semitendinoso, medialmente, projetando-se na altura da face caudal das articulações femoro tibial e femoro patelar - articulação do joelho, (Figura 1).

Os vasos arteriais destinados a esta estrutura derivam sempre, de ambos os lados, da artéria femoral caudal distal e variam de 10 a 1 (Tabela 1), com maior freqüência de 2 (7 vezes; $28 \%+/-9,0)$ à direita e de 6 a 2, igualmente com maior freqüência de 2 ( 8 vezes; $32 \%+/-9,3)$. Tão logo tem-se ainda à direita 3 ramos $(5$ vezes; $20 \%+/-8,0), 4(4 ; 16 \%+/-7,3), 5$ e 6 (3 vezes; $12 \%+/-6,5), 1,7$ e 10 ( 1 vez; $4 \%+/$ - 3,9). Ao que se refere ao linfonodo esquerdo, de mesma valia tem-se 3 (8 vezes; $32 \%+/-9,3), 4$ (6 vezes; $24 \%+/-8,5), 6(2$ vezes; $8 \%+/-5,4)$ e 5 ( 1 vez; $4 \%+/-3,9)$.

Relativamente as veias derivadas deste linfonodo, convergem sempre para a veia safena lateral, estes vasos oscilam entre 9 e 2 (Tabela 1), com maior freqüência de 3 (9 vezes; $36 \%+/-9,6)$, à direita e de 12 a 2 , com maior freqüência de 3 (10 vezes; $40 \%$ $+/-9,8)$ à esquerda. Assim, os demais ramos oriundos da linfonodo poplíteo direito e que se inserem na veia supracitada são em número de 2 e 5 ( 5 vezes; $20 \%+/-8,0 \%$ ), 4 e 6 ( 2 vezes; $8 \%+/-5,4)$ e 8 e 9 ( 1 vez; $4 \%$ $+/-3,9)$. Não obstante, ao lado esquerdo verifica-se em número de 2 ramos $(6$ vezes; $24 \%+/-8,5), 4$ (4 vezes; 16\% +/-7,3), 6 (3 vezes; $12 \%+/-6,5)$ e finalizando 9 e 12 ramos (1 vez; 4\%+/-3,9). Em ambos os casos, a inserção dos ramos arteriais bem 




Figura 1 - Face lateral do membro pelvino esquerdo do cão macho (obs. 16), mostrando a localização do linfonodo, entre os músculos bíceps femoral e semitendinoso, atrás das articulações femoro tibial e femoro patelar. Lf - linfonodo; SL - veia safena lateral; FCD r - ramo da artéria femoral caudal distal

Tabela 1 - Número de vasos arteriais e venosos relativos aos linfonodos poplíteos direito e esquerdo de cães Araçatuba - 2004

\begin{tabular}{ccccc}
\hline Vasos & \multicolumn{2}{c}{ artéria femoral caudal distal } & \multicolumn{2}{c}{ veia safena lateral } \\
\hline Obs & Ramos D & Ramos E & Ramos D & Ramos E \\
\hline $1(\mathrm{M})$ & 6 & 6 & 6 & 4 \\
$2(\mathrm{M})$ & 5 & 4 & 2 & 2 \\
$3(\mathrm{M})$ & 3 & 2 & 2 & 2 \\
$4(\mathrm{M})$ & 1 & 2 & 3 & 6 \\
$5(\mathrm{~F})$ & 2 & 2 & 3 & 3 \\
$6(\mathrm{M})$ & 3 & 4 & 5 & 9 \\
$7(\mathrm{~F})$ & 2 & 6 & 5 & 3 \\
$8(\mathrm{M})$ & 5 & 2 & 3 & 3 \\
$9(\mathrm{M})$ & 2 & 2 & 4 & 3 \\
$10(\mathrm{M})$ & 2 & 3 & 3 & 4 \\
$11(\mathrm{~F})$ & 2 & 2 & 5 & 6 \\
$12(\mathrm{M})$ & 3 & 3 & 9 & 12 \\
$13(\mathrm{~F})$ & 7 & 5 & 2 & 2 \\
$14(\mathrm{M})$ & 3 & 3 & 3 & 4 \\
$15(\mathrm{M})$ & 4 & 4 & 8 & 2 \\
$16(\mathrm{M})$ & 6 & 4 & 3 & 2 \\
$17(\mathrm{~F})$ & 10 & 3 & 2 & 3 \\
$18(\mathrm{M})$ & 6 & 2 & 6 & 6 \\
$19(\mathrm{M})$ & 5 & 4 & 5 & 4 \\
$20(\mathrm{M})$ & 4 & 4 & 5 & 3 \\
$21(\mathrm{~F})$ & 4 & 3 & 2 & 2 \\
$22(\mathrm{~F})$ & 3 & 3 & & 3 \\
\hline
\end{tabular}

D-direito e E-esquerdo, M- Macho, F- Fêmea

como a emergência dos ramos venosos ocorrem aleatoriamente, não existindo um local único ou coincidente entre as amostras. No que se refere ao tamanho e morfologia dos linfonodos poplíteos
(Tabela 2), pode-se verificar que as medidas do direito variam de $7,8 \mathrm{~mm}$ a $39,4 \mathrm{~mm}$ à medida dorso-ventral, $3,8 \mathrm{~mm}$ a $20,0 \mathrm{~mm}$ latero-lateral e $6,7 \mathrm{~mm}$ a $27,8 \mathrm{~mm}$ crâniocaudal, e mesmo modo as medidas do 
esquerdo, sendo $10,0 \mathrm{~mm}$ a $50,0 \mathrm{~mm}$ dorsoventral, $4,7 \mathrm{~mm}$ a $20,7 \mathrm{~mm}$ latero-lateral e $6,1 \mathrm{~mm}$ a $28,5 \mathrm{~mm}$ crânio-caudal, das quais observa-se a variação do forma destas estruturas de arredondada a alongada, em sua maioria com sutil achatamento laterolateral.

Uma característica encontrada neste estudo foi a presença de dois linfonodos num mesmo membro (Figura 2), ocorrendo em quatro dos 25 animais - que neste caso significam aproximadamente $16 \%$ do total - ora unilateralmente, em três exemplares, e em um exemplar ocorrendo nos dois membros pélvicos (Tabela 2). Tais órgãos, embora recobertos juntos pelo tecido adiposo que oferece sustentação aos mesmos, quando dissecados mostraram-se totalmente individualizados, cada qual com seus próprios ramos arteriais e venosos. $\mathrm{O}$ tamanho, forma e números de vasos entre linfonodos de um mesmo membro também variaram, não existindo necessariamente uma similaridade entre eles.

Dentro da amostra, foram observados ainda órgãos com sulco em sentido dorso-ventral ou crânio-caudal. Seis animais apresentam sulco em seus linfonodo direito, sendo que um deste cabe àqueles com dois linfonodos, e, também nesta situação, ambos mostram a característica. De mesmo modo, sete animais têm o sulco em seus linfonodos esquerdos. Assim, totalizam-se 10 cães com pelo menos um de seus linfonodos poplíteos apresentando tal característica, correspondendo a $45 \%$ da amostra (Tabela 2).

\section{Discussão}

Referente à bibliografia consultada, constata-se que há carência de informações, de cunho morfológico, relativas ao linfonodo poplíteo nos cães domésticos, sendo as

Tabela 2 - Medidas e descrição morfológica dos linfonodos poplíteo direito e esquerdo em cães - Araçatuba - 2004

\begin{tabular}{|c|c|c|c|c|c|c|c|c|c|}
\hline \multirow{3}{*}{ Obs } & & \multicolumn{3}{|c|}{ Linfonodo D } & \multicolumn{3}{|c|}{ Linfonodo E } & \multicolumn{2}{|c|}{ Sulco } \\
\hline & & DV & LL & $\mathrm{CC}$ & DV & LL & $\mathrm{CC}$ & $\mathrm{D}$ & $\mathrm{E}$ \\
\hline & & $(\mathrm{mm})$ & $(\mathrm{mm})$ & $(\mathrm{mm})$ & $(\mathrm{mm})$ & $(\mathrm{mm})$ & $(\mathrm{mm})$ & $(\mathrm{mm})$ & $(\mathrm{mm})$ \\
\hline $1(\mathrm{M})$ & $\mathrm{A}$ & 39,3 & 11,6 & 22,7 & 50,0 & 13,7 & 26,6 & + & + \\
\hline $2(\mathrm{M})$ & A & 16,5 & 8,7 & 15,1 & 20,5 & 11,5 & 10,1 & - & - \\
\hline $3(\mathrm{M})$ & A & 19,9 & 11,6 & 10,3 & 18,6 & 13,6 & 7,0 & - & - \\
\hline $4(\mathrm{M})$ & A & 14,5 & 8,2 & 8,4 & 13,4 & 8,9 & 8,8 & - & - \\
\hline $5(\mathrm{~F})$ & A & 24,0 & 8,8 & 13,4 & 27,4 & 7,2 & 18,0 & - & - \\
\hline $6(\mathrm{M})$ & A & 24,4 & 8,3 & 14,7 & 22,7 & 13,3 & 6,1 & - & - \\
\hline \multirow{2}{*}{$7(\mathrm{~F})$} & A & 38,4 & 12,2 & 27,8 & 31,1 & 12,0 & 28,5 & - & - \\
\hline & B & I & I & 1 & 30,1 & 12,4 & 19,2 & l & - \\
\hline $8(\mathrm{M})$ & A & 22,8 & 13,2 & 9,6 & 22,6 & 9,6 & 11,9 & + & + \\
\hline $9(\mathrm{M})$ & A & 22,7 & 20,0 & 12,5 & 25,9 & 20,7 & 9,4 & - & + \\
\hline $10(\mathrm{M})$ & A & 32,1 & 10,2 & 13,8 & 25,8 & 4,7 & 8,3 & + & - \\
\hline $11(F)$ & A & 20,1 & 6,0 & 9,9 & 21,0 & 6,1 & 14,9 & - & - \\
\hline $12(\mathrm{M})$ & A & 37,2 & 11,7 & 20,7 & 39,5 & 11,2 & 20,4 & + & + \\
\hline \multirow{2}{*}{$13(F)$} & A & 23,5 & 7,6 & 16,6 & 21,9 & 7,8 & 15,3 & - & - \\
\hline & B & 7,8 & 7,1 & 7,4 & 10,0 & 9,5 & 10,1 & - & - \\
\hline $14(\mathrm{M})$ & A & 20,8 & 11,0 & 12,5 & 20,2 & 9,6 & 11,6 & - & - \\
\hline $15(\mathrm{M})$ & A & 30,1 & 7,0 & 25,5 & 27,5 & 6,9 & 22,2 & + & + \\
\hline \multirow{2}{*}{$16(\mathrm{M})$} & A & 31,5 & 6,8 & 19,0 & 48,8 & 12,2 & 21,6 & + & + \\
\hline & B & 28,8 & 10,8 & 20,6 & I & 1 & I & + & 1 \\
\hline \multirow{2}{*}{$17(\mathrm{~F})$} & A & 8,7 & 3,8 & 6,7 & 24,5 & 6,6 & 9,0 & - & + \\
\hline & B & 15,0 & 5,3 & 8,8 & I & I & I & - & I \\
\hline $18(\mathrm{M})$ & $\mathrm{A}$ & 18,5 & 5,4 & 9,0 & 28,0 & 6,4 & 6,8 & - & + \\
\hline $19(\mathrm{M})$ & A & 39,4 & 14,5 & 24,5 & 40,4 & 14,0 & 15,8 & - & - \\
\hline $20(\mathrm{M})$ & A & 19,0 & 9,1 & 10,3 & 18,2 & 11,4 & 11,4 & - & - \\
\hline $21(\mathrm{~F})$ & A & 23,0 & 11,8 & 10,0 & 19,0 & 10,7 & 8,5 & - & - \\
\hline $22(\mathrm{~F})$ & A & 19,4 & 7,2 & 12,6 & 15,2 & 12,2 & 10,9 & - & - \\
\hline
\end{tabular}

JV-dorso-ventral, LL-latero-lateral, CC-crânio-caudal, D-direito, E-esquerdo, $(+)$-presença de sulco, $(-)$-ausência de sulc M-Macho, F- Fêmea, A e B referem-se ao(s) linfonodo(s) 


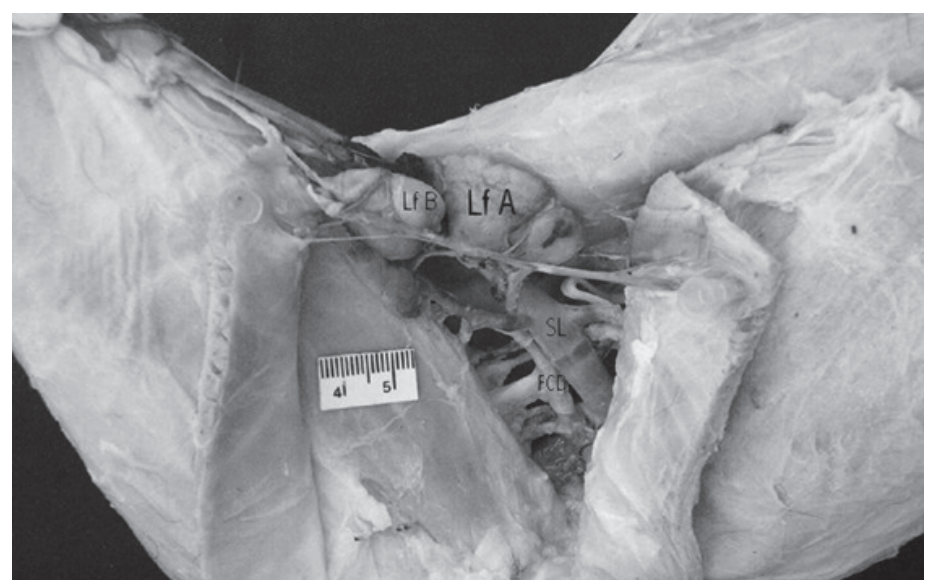

Figura 2 - Face lateral do membro pelvino direito do cão macho (obs. 16) onde é possível visualizar dois linfonodos - Lf A,cranial e Lf B, caudal - Observa-se os vasos a femoral caudal distal - FCD- e v. safena lateral - SLemitindo e recebendo, respectivamente, ramos dos linfonodos independentemente

informações de caráter genérico.

Em concordância com alguns autores $^{1,2,4,7,13,14}$ pode-se observar que os linfonodos poplíteo nos cães apresentamse na forma arredondada ou ovalada, com 2 a $3 \mathrm{~cm}$ de diâmetro, em média. Localizam$\mathrm{se}$, ainda, na fossa poplítea sobre o $\mathrm{m}$. gastrocnêmio e entre o $\mathrm{m}$. bíceps femoral, medialmente, e m. semitendinoso, lateralmente; envolto por tecido adiposo e inserido no subcutâneo.

Embora Budras, Fricke e McCarthy ${ }^{12}$ tenham representado esquematicamente as veias do linfonodo, em seu texto nada informa a respeito dos vasos sanguíneos. Cordeiro e Baracat ${ }^{4}$ sugerem que linfonodos maiores têm um hilo pelo qual emergem seus eferentes e transitam seus vasos sangüíneos nutrícios. Discordando deste autor, Spalding e Heath ${ }^{7}$ e Belz e Heath ${ }^{14}$ estudando, respectivamente, suínos e cães notaram a ausência do hilo e presença de uma rede vascular, assemelhando-se sobremaneira com as observações do presente trabalho.

Os vasos sangüíneos arteriais que promovem a nutrição dos linfonodos poplíteos nos cães derivam da artéria femoral caudal distal, independentemente do número de ramos existentes. Da mesma forma, as veias emergentes de tal estrutura dirigem-se a um único vaso sangüíneo venoso, a veia safena lateral. Ainda conforme resultados obtidos, tais ramos, sejam estes arteriais ou venosos, não apresentam um local único de inserção ou emergência, ocorrendo estes aleatoriamente ao órgão, indo, portanto, de encontro às observações de Salvador, Pereira e Grande ${ }^{9}$ e Salvador et al. ${ }^{10}$ em relação ao linfonodo traqueobraquial em cães e dos dois últimos autores.

Outro fato constatado e relevante nos resultados foi a presença de sulcos em vários dos linfonodos, bem como a existência de dois linfonodos em um mesmo membro pélvico, totalmente individualizados e com vascularização, arterial e venosa, próprias.

Considerando-se que este estudo tenha sido realizado com animais soropositivos para Leishmaniose Visceral e que tal enfermidade mostra-se, por vezes, através da linfoadenomegalia, é preciso cautela ao relacionar o tamanho do órgão ao número de ramos dos vasos, tendenciando -se a acreditar que o aumento na ocorrência destes vasos esteja correlacionado à hipertrofia e à hiperplasia do órgão. De igual consideração deve ser o fato da amostra ser composta por animais de diferentes raças e, portanto, diferentes portes físicos, mascarando, também, quaisquer suposições desta ordem.

Todavia, a presença dos sulcos e doa "linfonodos duplos" notado num considerável percentual da amostra permite a sugestão de uma progressiva organização 
destes nodos linfáticos acompanhado de angiogênese, paralelamente à sua intensa hipertrofia em animais acometidos pela enfermidade.

\section{Conclusão}

Frente aos resultados obtidos no presente estudo e com base na literatura atual, pode-se concluir que os linfonodos poplíteos nos cães, à direita e à esquerda, localizam-se entre os músculos bíceps femoral e semitendinoso, superficialmente e sendo facilmente palpáveis.

Tais estruturas recebem vasos arteriais oriundos da artéria femoral caudal distal e emitem vasos venosos que desembocam na veia safena lateral, podendo o número de ramificações destes e daqueles variar entre $\mathrm{o}$ nodo direito e o esquerdo.

O tamanho do linfonodo poplíteo em cães soropositivos para Leishmaniose Visceral pode encontrar-se entre 7,8 x 3,8 8 6,1 a $50,0 \times 20,7 \times 28,5$, em mm, sendo a média $26,18 \times 10,5 \times 15,97 \mathrm{~mm}$ para o direito e $26,98 \times 11,14 \times 15,25 \mathrm{~mm}$ para o esquerdo (referente as medidas dorso-ventral, laterolateral e crânio-caudal, respectivamente).

Dados conclusivos a respeito dos achados $3 / 4$ sulcos e "linfonodos duplos" $3 / 4$ assim como da existência de correlação entre o tamanho do linfonodo e número de vasos sangüíneos necessitam outros estudos em continuidade a esta pesquisa.

\section{Arterial and venous vasculature of popliteo lymph nodes in dogs}

\section{Abstract}

Poplíteo lympho nodes in the dogs is placed, to the right and the left in popliteo an appointed space fossa, in distal part of the muscles femoral biceps, laterally and semi-tendinous, medially, projecting in the height of the face volume of the joint to femoro tibial e femoro patellar (joint of the knee). In this study twenty and five dogs, males and females adult, originating the Araçatuba municipal kennel had been used whose captures had been effected by the animal sanitary defense of this city. The arterial vessels destined to this structure always derive, of both the sides, the femoral artery distal volume and vary of 10 the 1 , more frequently of 2 (7 times, $28 \%$ ) to the right and of 6 the 2 , equally more frequently of 2 (8 times, $32 \%$ ). So soon as one has still to right 5 and 6 branches ( 3 times, $12 \%), 1,7$ and 10 (1 time, 4\%). Relatively to the veins derived from this lympho nodes, always converges to the lateral safena vein, these vessels oscillates between 9 and 2, more frequently of 3 (6 times, $24 \%$ ), to the right and of 12 the 2 , more frequently of 3 (10 times, $40 \%$ ) to the left. Thus, others deriving branches of popliteo lympho nodes right and that if they insert in the above-mentioned vein are in number of 2 and 5 ( 5 times, 20\%), 4 and 6 (2 times, $8 \%$ ) and 8 and $9(1$ time, $4 \%$ ). In spite of, to the left side it is examined in number de 2 branches (6 times, 24\%), 4 (4 times, 16\%), 6 (3 times, 12\%) and finishing 9 and 12 branches (1 time, $4 \%$ ). The size of popliteo lympho node in seropositive dogs for Visceral Leishmaniasis can meets enters 7,8 x 3,8 x 6,1 a 50,0 x $20,7 \times 28,5$, in mm, being average 26,18 x 10,5 x 15,97 mm for right and $26,98 \times 11,14 \times 15,25 \mathrm{~mm}$ for left (concerning the measures dorsoventral, latero-lateral and cranial-caudal, respectively).

\section{Referências}

1 GETTY, R. Anatomia dos animais domésticos de Sisson \& Grossman. 5. ed. Rio de Janeiro: Guanabara, 1981.
Key words:

Dogs.

Popliteo lympho nodes. Anatomy.

Vasculature.

\author{
2 PFLUG, J. J.; CALNAN, J. S. Lymphatics: Normal \\ anatomy in the dog hind leg. Journal of Anatomy, $v$. \\ 105, p. 457-465, 1996.
}

3 SCHWARZE, E.Compendio de anatomia veterinária. 
1. ed. Zaragoza: Acribia, 1972. p. 120

4 CORDEIRO, A. K.; BARACAT, F. F. Linfologia. São Paulo: Fundo Editorial BYK-PROCIENX, 1983. cap 3, p. 12-17.

5 KNOX, P.; PFLUG, J. J.The effect of the canine popliteal node on the composition of lymph. Journal of Physiology, v. 345, p. 1-14, 1983.

6 PARSONS, T. S.; ROMER, A. S. Anatomia comparada dos vertebrados. São Paulo: Atheneu, 1985. p. 388390.

7 SPALDING, H. J.; HEATH, T. J. Blood vessels of lymph nodes in the pig. Research Veterinary Science, v. 41, pt. 2, p. 196-199, 1986.

8 HALL, J. G.; SINNET, H. D. The functioning in unanaesthetized sheep of the popliteal lymph node after the surgical removal of its blood supply. Brazilian Journal of Experimenthal Pathology, v. 68, pt. 1, p. 125-132, 1987.

9 SALVADOR, A. C.; PEREIRA, A. S.; GRANDE, N. R. Microanatomy of the blood vasculature of lymph node follicles in the dog. Lymphology, v. 25, pt. 1, p. 37-41,
1992.

10 SALVADOR, A. C.; PEREIRA, A. S.; DE SÁ, C. M.; GRANDE, N .R. Blood vasculature of the lymph node in the dog: anatomical evidence for participation of extrahilar arterial vessels in the blood suplly of the cortex. Acta Anatomic (Basel), v. 143, pt. 1, p. 41-47, 1992.

11 EVANS, H. E. Miller's anatomy of the dog. 3. ed. Philadelphia: W.B. Saunders Company, 1993. p. 748.

12 BUDRAS, K. D.; FRICKE, W.; MCCARTHY, P. H. Anatomy of the dog. 3. ed. Alemanha: Mosby-Wolfed, 1994. cap 7.

13 SASAKI, K.; PABST, R.; ROTHKOTTER, H. J. Development of the high endothelial venule in rat lymph node autografts. Anatomical Record, v. 238, pt. 4, p. 473-479, 1994.

14 BELZ, G. T.; HEATH, T. J. Pathways of blood flow to and through superficial lymph nodes in the dog. Journal of Anatomy, v. 187, pt. 2, p. 413-421, 1995.

15 BARONE, R. Anatomie comparée des mammifères domestiques. Paris: Vigot, 1996. v. 5, 832 p. 